\title{
Improvement suggestions for problems of hazard map from the viewpoint of color scheme
}

\author{
Ryo ENDO $^{\text {a, } * \text {, Takayuki NAKANO }}{ }^{\text {a }}$ \\ ${ }^{a}$ Geospatial Information Authority of Japan; endoh-r96pc@mlit.go.jp, nakano-t96fj@ mlit.go.jp \\ * Corresponding author
}

\begin{abstract}
Local governments often rely on hazard maps to plan for and respond to local natural disasters. These maps often rely on the use of many colors, but their exact color scheme and style differs in each area. As a result, we hypothesize that some users, specifically colorblind individuals, may have difficulty correctly understanding the information on some of these hazard maps. In this study, we test that hypothesis by conducting a survey of Japanese liquefaction hazard maps and their visual accessibility. To this end, we first undergo a survey of the color schemes used in these maps and investigate whether they are easily understood by people with colorblindness. We next specifically analyze several maps we deem particularly problematic in terms of color scheme and visibility, using these as case studies to discuss issues with accessibility and to summarize possible countermeasures. Our survey found that liquefaction hazard maps use one of three main color schemes: "diverging color scheme," "Sequential cold colors," or "sequential warm colors." However, while there were issues with several maps, including difficulty reading the background map or correctly understanding the risk of liquefaction, these difficulties were not related to the color scheme used. To improve the accessibility of hazard maps, therefore, is necessary to create a unified manual that contains the following information: an examination of colors, the utilization of a universal design check tool, and the use of GIS vector data.
\end{abstract}

Keywords: universal design, hazard map, color scheme, colorblindness, accessibility

\section{Introduction}

Hazard maps are often used by local governments and residents both to help take necessary measures against disasters and to effectively respond to them once they occur. Such maps frequently use colors to illustrate relative risk or to make the map stand out, a phenomenon that has become even more common with the development of computer technology and growing ubiquitous nature of color printing (Ito, 2012). Such highly visible hazard maps can help users interpret them in their disaster prevention measures and/or during of a disaster.

In Japan, each local government is responsible for developing its own hazard maps, meaning that their elements, including color schemes, are not always uniform. In addition, the more information a hazard map includes (i.e., hazard risk level, background diagram, location of shelters, and/or adaptive evacuation routes), so the more colors it is likely to use. This may cause reduced visibility for some users, especially for people with colorblindness, who may difficulty in distinguishing specific colors. Such people occupy a sizable minority of the population (4-8\% of men, $0.5 \%$ of women; Okabe and Ito, 2008), and ensuring these individuals can accurately read and interpret hazard maps is importantin the case of disaster.
To examine how to solve this issue, in this study we use liquefaction hazard maps as a case study to examine the coloring and design of published hazard maps in Japan. Liquefaction hazard map shows liquefaction susceptibility or its damage level in a specific area. After conducting a survey of these maps, we examine a few specific examples and highlight their accessibility issues. We then use these examples to highlight some possible solutions.

\section{Understanding colorblindness}

Human visual ability is dictated by three different genes, each of which controls the production of different kinds of cells that detect light. The L-cone opsin gene creates L-cones, which detect long wavelength light, while the production of M-cones, which detect intermediate wavelengths and S-cones, which detect short wavelength light, are controlled by their own respective genes. There are five types of vision, depending on whether individuals are missing one of these genes (e.g. Ito, 2012):

1) Common type (C-type): People who have all three types of cone opsin genes

2) Protanopia (P-type, red-colorblindness): People lacking the L-cone opsin gene or people whose L-cone opsin gene is replaced by an intermediate one between L- and M-cone opsin genes

3) Deuternopia (D-type. green-color blindness): People lacking the M-cone opsin gene or people 
whose M-cone opsin gene is replaced by an intermediate one between L- and M-cone opsin genes.

4) Tritanopia (T-type, blue-color blindness): People lacking the S-cone opsin gene

P-type and D-type are often called red-green colorblindness and is harder to distinguish red and green than C-type. T-type is often called blue-color blindness and is harder to distinguish blue colors than C-type.

Of the color vision abnormalities, red-green colorblindness is the most common: This study focuses on red-green colorblindness.

\section{Methods}

As of November 13, 2018, ground damage (liquefaction) maps have been published by 366 district municipalities in Japan (Ministry of Land, Infrastructure and Transport, 2018). From July to September 2016, we were able to access 266 of these maps for our study. For each map, we verified whether color universal design was taken into consideration and whether visibility is ensured using the following method.

\subsection{Color scheme}

There are no objective criteria for evaluating the visibility of a hazard map. The large number of available hazard maps makes it impossible to use questionnaire surveys or other qualitative methods. Therefore, we quantitatively evaluated each map using colorblind proofing software, Adobe Photoshop CC 2018.

\subsubsection{Number of color classes}

For each map, we determined the total number of color classes based on the number of liquefaction risk categories in the legend. If the map contained other colored areas that were neither color-coded as a liquefaction risk nor specified in the legend, those areas were counted together as one color.

\subsubsection{Color-blind simulation and colorimetry}

We then used Touyama (2005) to determine whether liquefaction risk, based on a map's legend, could be correctly identified by a person with colorblindness.

First, using Photoshop, we converted the original hazard map images to P-type and D-type simulation images. We then measured each color representing liquefaction risk in the map's legend. We adopted two models to determine colorimetry for calculating color difference as shown in 2.1.3 and grasping what kind of colors are used in liquefaction hazard maps as shown in 2.1.4.. The first, the $\mathrm{L} * \mathrm{a} * \mathrm{~b} *$ color model, includes $L^{*}$ (luminance), $a^{*}$ (redness), and $b^{*}$ (yellowness). We measured the $L^{*} a^{*} b^{*}$ values for C-, P- and D-type colorblindness for all maps. Second, the HSB color model includes $H$ (hue), $S$ (saturation), and $B$ (brightness), and we measured $H S B$ values for C-type colorblindness. We conducted each measurement five times and adopted the average value. Colorimetry was not performed when the hazard map images used hatch-type shading and so on.

\subsubsection{Calculating color difference}

Using our results from the previous section, we then calculated the color difference of the legends using the CIEDE 2000 formula $\left(\Delta E_{00}\right)$ color difference model proposed by CIE (Commission internationale de l'éclairage) (Luo et al., 2001). CIEDE 2000 is considered the color difference model consistent with subjective vision (e.g. Yang et al., 2012). Using the following formula results in an index that allows us to numerically express color difference (Sharma et al., 2005):

$\Delta E_{00}=\sqrt{\left(\frac{\Delta L^{\prime}}{k_{L} S_{L}}\right)^{2}+\left(\frac{\Delta C^{\prime}}{k_{C} S_{C}}\right)^{2}+\left(\frac{\Delta H^{\prime}}{k_{H} S_{H}}\right)^{2}+R_{T} \frac{\Delta C^{\prime}}{k_{C} S_{C}} \frac{\Delta H^{\prime}}{k_{H} S_{H}}}$

where $\Delta L^{\prime}=$ difference of lightness and function of $L^{*}$

$\Delta C^{\prime}=$ difference of chroma and function of $a^{*}$ and $b^{*}$

$\Delta H^{\prime}=$ difference of hue and function of $a^{*}$ and $b^{*}$

$R_{T}=$ rotation function corresponding to blue color difference calculation

$S_{L}, S_{c}$ and $S_{H}=$ scaling functions

$k_{L}, k_{c}$ and $k_{H}=$ weighting parameters for lightness, chroma, and hue, respectively. These parameters are normally set to 1 .

To judge whether map users can interpret the liquefaction risk in the legend, we set the threshold value of the color difference $\Delta E_{00}$, which can distinguish two arbitrary colors out of liquefaction risk classification map legends, to 10 (Alžběta and Arzu, 2017).

\subsubsection{Characteristic analysis of color scheme}

We then analyzed the characteristics of hazard maps' color schemes. First, based on the saturation value $(S)$ obtained in Section 2.1.2, colors were classified as chromatic $(S \geq 5)$ or achromatic $(S<5)$. Using the hue value $(H)$, we then classified chromatic color into six additional categories: red, yellow, green, cyan, blue, and purple.

\begin{tabular}{ll}
\hline Hue & Color \\
\hline $330<H<360 ; 0 \leq H \leq 30$ & Red \\
$30<H \leq 90$ & Yellow \\
$90<H \leq 150$ & Green \\
$150<H \leq 210$ & Cyan \\
$225<H \leq 270$ & Blue \\
$270<H \leq 330$ & Purple \\
\hline
\end{tabular}

Table 1. Classification of chromatic colors based on hue

Finally, we carried out a cluster analysis and analyzed trends in color combinations in liquefaction hazard maps. We used the SPSS Statistics 24.0 (IBM) two-step cluster analysis tool. The number of clusters was set to 5 . 


\subsection{Survey of hazard map visibility}

After observing the original hazard maps and comparing them to the simulation images created in Section 2.1.2, we extracted maps we found problematic or inaccessible from the perspective of visibility. Reasons for these issues included readability of map contents, difficulty discriminating between legend colors and other colors on the map, and other elements in addition to color schemes. In addition, these case studies include both paper and electronic maps.

\section{Results}

\subsection{Color scheme}

\subsubsection{Number of color classes}

Figure 1 shows the number of colors used in each liquefaction hazard map included in our sample size. Over $90 \%$ of maps studies displayed risk level using color classes; these ranged from 2 colors to 9. Most maps used 4 color classes, followed by maps using 5 and then 3: together these three groups accounted for $80 \%$ of all hazard maps. However, $5 \%$ of hazard maps did not rely on color to demonstrate risk. For example, some maps illustrated regions at risk of liquefaction by surrounding them with a line and/or covering the area with hatch marks. None of the maps studies relied on gradations or shading.

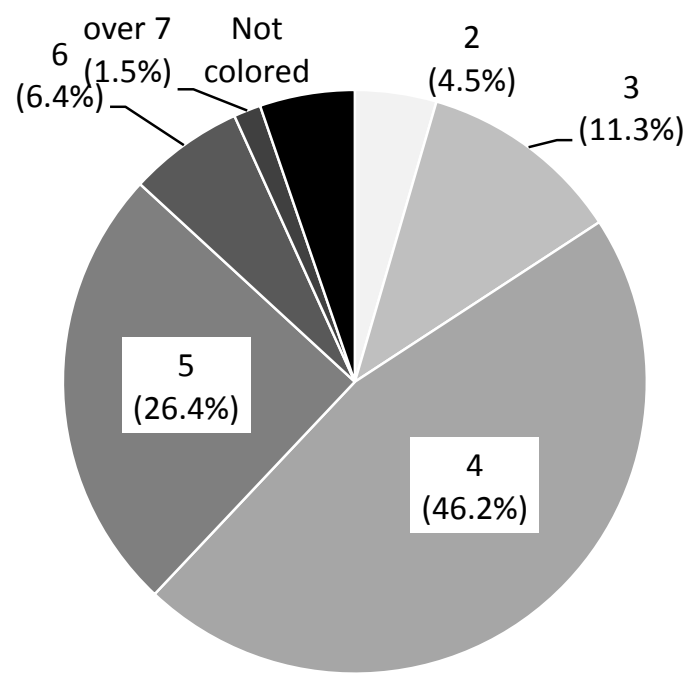

Figure 1. The number of color classes used to express degree of risk in Japanese liquefaction hazard maps.

\subsubsection{Measuring $\Delta E_{00}$}

About $60 \%$ of the maps studies had a minimum $\Delta E_{00}$ value below the threshold of 10 when examining the simulation images of C-, P- and D-type colorblindness (Figure 2). As the number of colors increases, the proportion of hazard map where the minimum value of the color difference is below the threshold value increases as well.

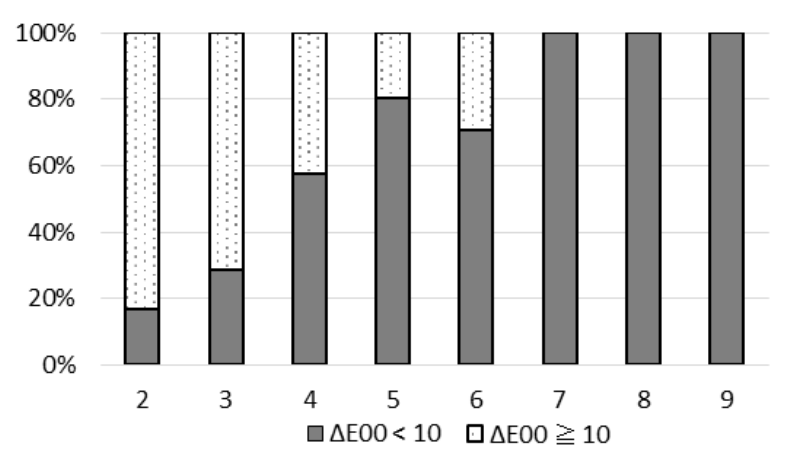

Figure 2. Percentage of each map where the minimum value of the color difference, $\Delta E_{00}$, is less than the threshold value 10 .

\subsubsection{Classification of color schemes}

For the purposes of this study, we focused our research on maps containing 4 color classes, since these made up the largest proportion of the total. Table 2 shows the results of our cluster analysis performed on 123 maps with 4 color classes, which analyzed the color schemes used. Degree of risk is relative, because the expression and meaning of risk differ depending on the hazard map: 4 means relatively high risk value and 1 is relatively low risk. Since each color includes various hue values as shown in Table 1, it is necessary to note that even the same color name indicates different colors in Table 2.

Cluster 1 consists of maps with red-type, yellow-type, green-type, and achromatic color classes, which account for $20.3 \%$ of the total maps. Cluster 2 (14.6\%) includes red-type colors in the degree of risk 4 to 2 , and achromatic color classes in the degree of risk 1; Cluster 3 $(30.9 \%)$ includes red-type color class in the degree of 4 , yellow-type color classes in the degree of risk 3 and 2, and achromatic color class in the degree of risk 1; Cluster $4(11.4 \%)$ includes cyan-type color classes in the degree of risk 4 to 2, and achromatic color class in the degree of risk 1; and Cluster 5 includes red-type color class in the degree of risk 4, yellow-type color classes in the degree of risk 3 to 2, and cyan-type color class in the degree of risk 1. In Clusters 4 and 5, the proportion of the map with a minimum $\Delta E_{00}$ less than 10 was particularly high $(\sim 80 \%)$.

Based on this analysis, we classified hazard maps into three types, using Brewer (1994) to label them: Clusters 1 and 5 are "diverging color schemes" $(\mathrm{N}=53,43.1 \%)$ and include red-, yellow-, and green-/cyan- type color classes. Clusters 2 and 3 can be understood as "sequential warm color schemes" ( $\mathrm{N}=56,45.5 \%)$ and include red- and yellow- type color is a "sequential cold color scheme" (N $=14,11.4 \%$ ) and includes cyan-type color classes. 


\begin{tabular}{|c|c|c|c|c|c|}
\hline \multirow{2}{*}{$\begin{array}{l}\text { Degree } \\
\text { of risk }\end{array}$} & \multicolumn{5}{|c|}{ Cluster } \\
\hline & 1 & 2 & 3 & 4 & 5 \\
\hline & $\begin{array}{l}\mathrm{N}=25 \\
(20.3 \%)\end{array}$ & $\begin{array}{l}\mathrm{N}=18 \\
(14.6 \%)\end{array}$ & $\begin{array}{l}\mathrm{N}=38 \\
(30.9 \%)\end{array}$ & $\begin{array}{l}\mathrm{N}=14 \\
(11.4 \%)\end{array}$ & $\begin{array}{l}\mathrm{N}=28 \\
(22.8 \%)\end{array}$ \\
\hline 4 (high) & $\begin{array}{l}\mathrm{R} \\
(96.0 \%)\end{array}$ & $\begin{array}{l}\mathrm{R} \\
(66.7 \%)\end{array}$ & $\begin{array}{l}\mathrm{R} \\
(63.2 \%)\end{array}$ & $\begin{array}{l}\mathrm{C} \\
(46.7 \%)\end{array}$ & $\begin{array}{l}\mathrm{R} \\
(96.4 \%)\end{array}$ \\
\hline 3 & $\begin{array}{l}Y \\
(100 \%)\end{array}$ & $\begin{array}{l}\mathrm{R} \\
(88.9 \%)\end{array}$ & $\begin{array}{l}\text { Y } \\
(89.5 \%)\end{array}$ & $\begin{array}{l}\mathrm{C} \\
(64.3 \%)\end{array}$ & $\begin{array}{l}Y \\
(67.9 \%)\end{array}$ \\
\hline 2 & $\begin{array}{l}\text { G } \\
(100 \%)\end{array}$ & $\begin{array}{l}\mathrm{R} \\
(61.1 \%)\end{array}$ & $\begin{array}{l}Y \\
(39.5 \%)\end{array}$ & $\begin{array}{l}\mathrm{C} \\
(64.3 \%)\end{array}$ & $\begin{array}{l}Y \\
(67.9 \%)\end{array}$ \\
\hline 1 (low) & $\begin{array}{l}\text { A } \\
(100 \%)\end{array}$ & $\begin{array}{l}\text { A } \\
(94.4 \%)\end{array}$ & $\begin{array}{l}\text { A } \\
(97.4 \%)\end{array}$ & $\begin{array}{l}\text { A } \\
(71.4 \%)\end{array}$ & $\begin{array}{l}\mathrm{C} \\
(39.3 \%)\end{array}$ \\
\hline $\begin{array}{l}\Delta E_{00}< \\
10\end{array}$ & $\begin{array}{l}16 \\
(64 \%)\end{array}$ & $\begin{array}{l}10 \\
(52.6 \%)\end{array}$ & $\begin{array}{l}21 \\
(55.2 \%)\end{array}$ & $\begin{array}{l}11 \\
(78.6 \%)\end{array}$ & $\begin{array}{l}24 \\
(85.7 \%)\end{array}$ \\
\hline
\end{tabular}

Table 2. Result of cluster analysis. $R, Y, G$, and $C$ represent colors of red, yellow, green, and cyan respectively, and A represents achromatic colors. The most frequent color is displayed in each cell.

\subsection{Accessibility issues}

\subsubsection{Hard to recognize map background or characters}

Many hazard maps include a background map. However, it is sometimes difficult to read the background image or the characters on it; it may be blurry due to scanning or the colors used in communicating risk cover it (Figure 3 ).

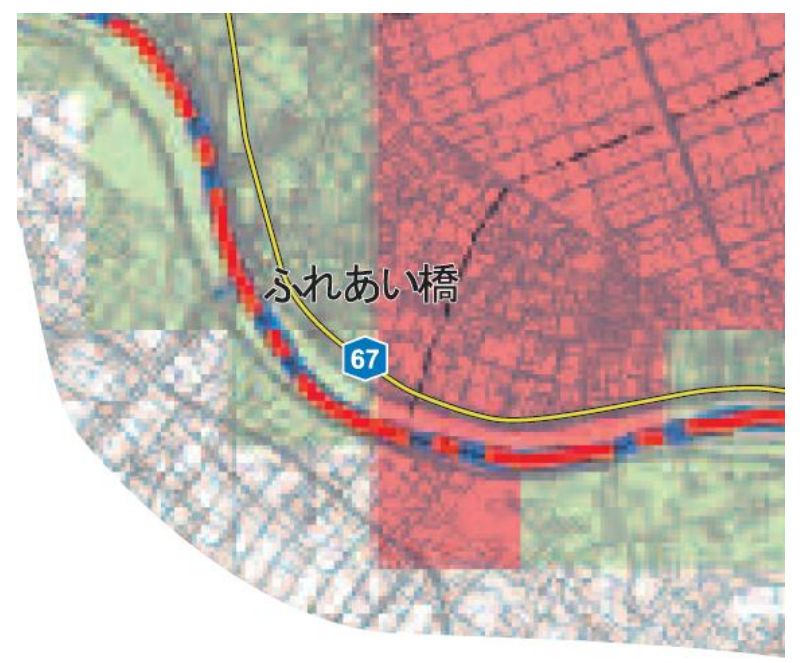

Figure 3. Example of a map where the background is blurry and illegible, with the exception of the posted name and the road, both added subsequently.

\subsubsection{Legend and map include different colors}

There were also some examples where a map's color coding did not agree with the colors used in the legend. For example, in Figure 4, according to the legend areas with a low risk of liquefaction should be marked in gray. However, this color does not appear on the map; perhaps it corresponds with the light blue area. We found other examples where the legend was clearer than the map or where the legend colors differed slightly from those used in the map. It is possible that in these examples the legend was created after scanning a preexisting map.

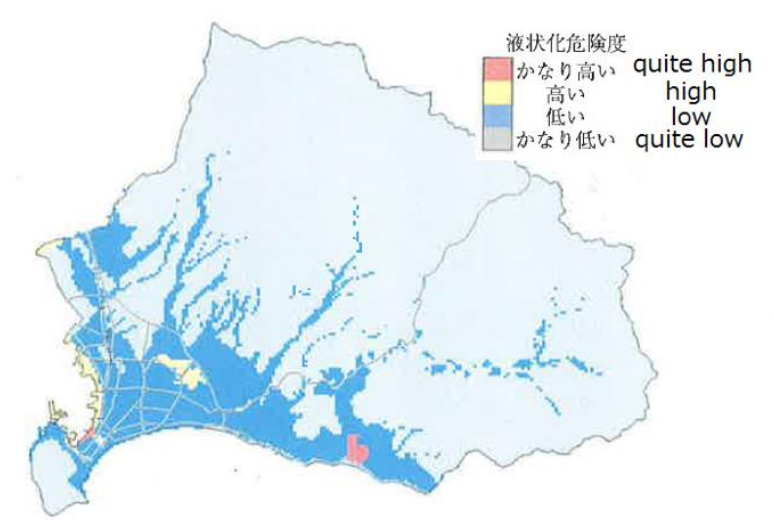

Figure 4. Example of a map where the legend colors differ from those used in the map itself. Areas of "quite low" risk are marked in gray on the legend, but seem to be illustrated in light blue on the map.

\subsubsection{Confusing use of hatching and background}

Instead using color to designate danger level, some maps rely on hatching. While illustrating risk information without color may help a map be more accessible, according to color universal design (Okabe and Ito, 2002), in some cases the hatching overlapped with the background map, making it difficult to read (Figure 5).

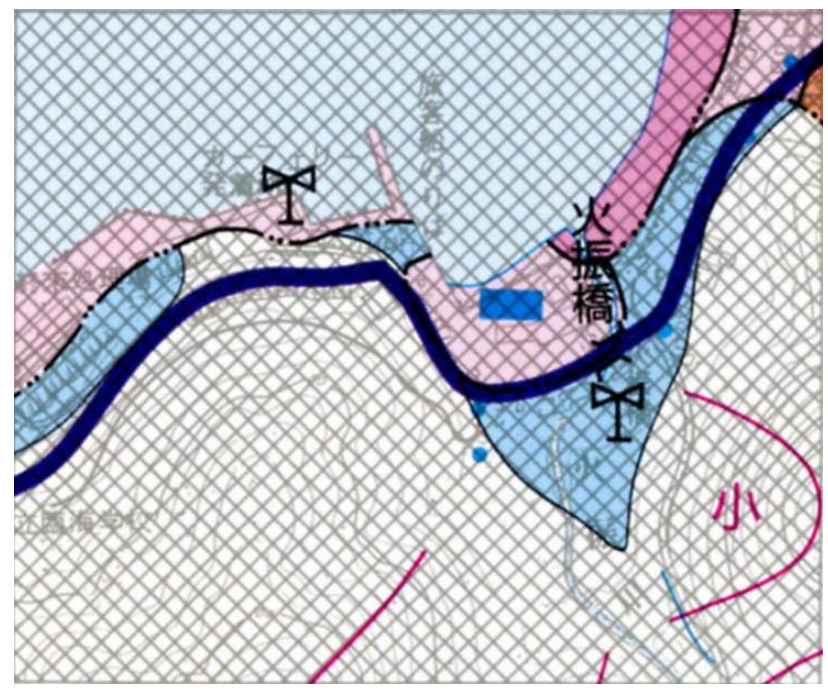

Figure 5. Example in which overlapping shading and background make a map difficult to read. The gray hatched area represents areas at risk of liquefaction, but the lines are the same color as the characters and contour lines of the background map, making it hard to read accurately.

4.2.4 Legend colors easily confused with other map colors

In other cases, the map used colors to portray liquefaction risk color that could easily be confused with the colors used in other map features, such as water or roads (Figure $6)$, especially for people with colorblindness.

\subsubsection{Inconsistent legend arrangement}

While most maps' legends list risk levels from high to low, some go from low to high, making it difficult to accurately interpret unless a user is paying close attention (Figure 6). 


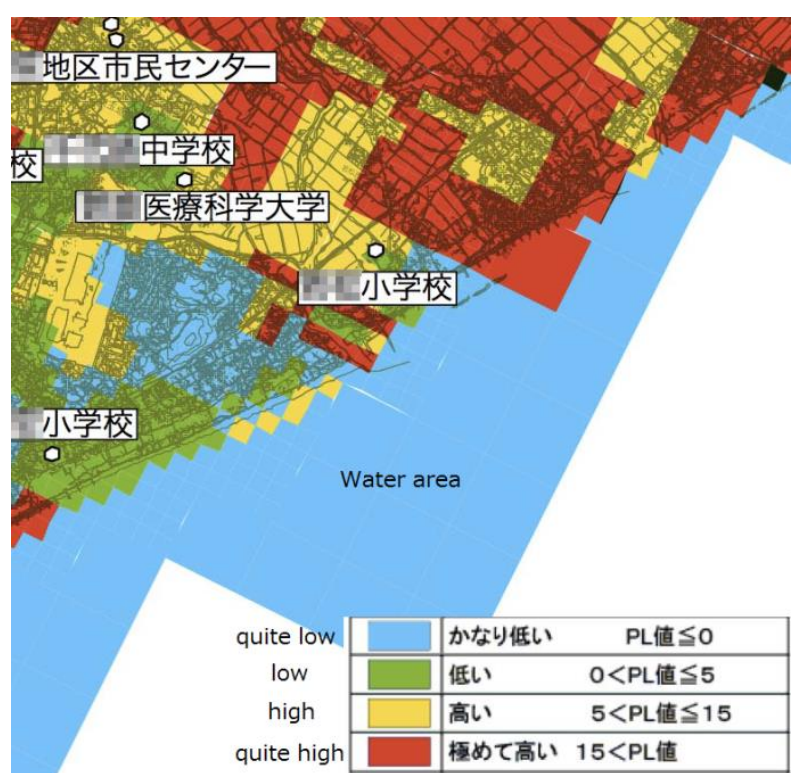

Figure 6. Example where we cannot distinguish the color class "quite low" and that of water area. This is also the example which expresses higher risk level on the lower side of the legend

4.2.6 Similar colors used to portray liquefaction risk and other risk information

Another example of a confusing map is where the colors used to express liquefaction risk are similar to those used to express other risk, including sediment-related disasters, flood damage information, etc., making it difficult to correctly recognize the risk of liquefaction (Figure 7).

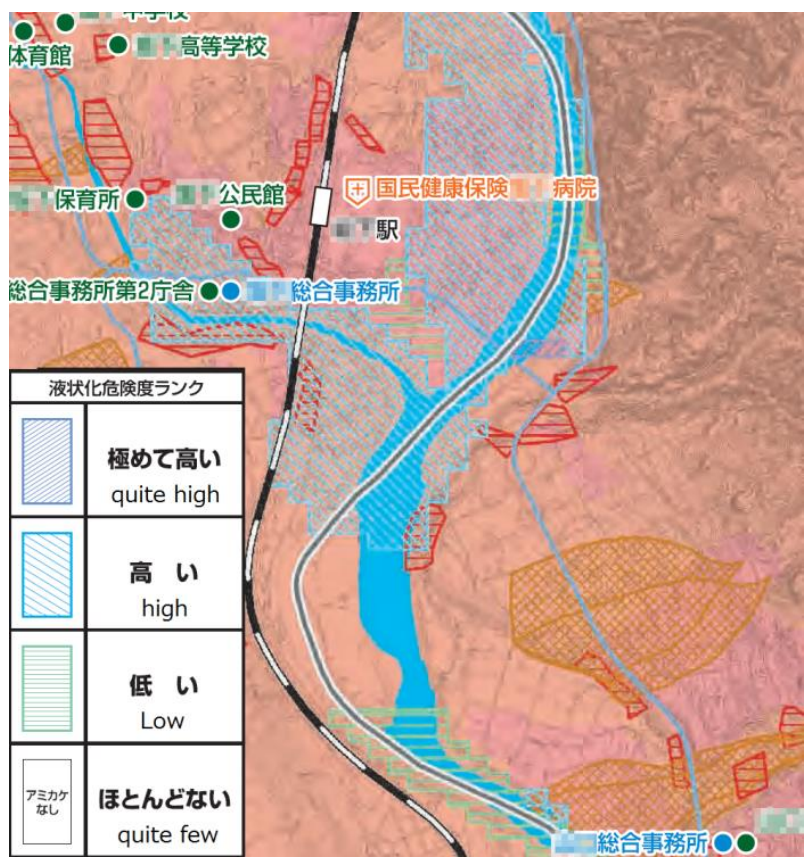

Figure 7. Example in which colors used to represent liquefaction risk overlap with those representing other risk information. The light blue and yellow-green shades indicate areas at risk of liquefaction.

\subsubsection{Use of too many color classes}

There were some maps that expressed degree of risk based on the $P_{L}$ value, an evaluation index of liquefaction (Figure 8). One such map divided its legend into 9 classes, making even the original map difficult to understand. Such a map is especially troublesome for people with colorblindness when attempting to quickly understand the differences in risk classification.

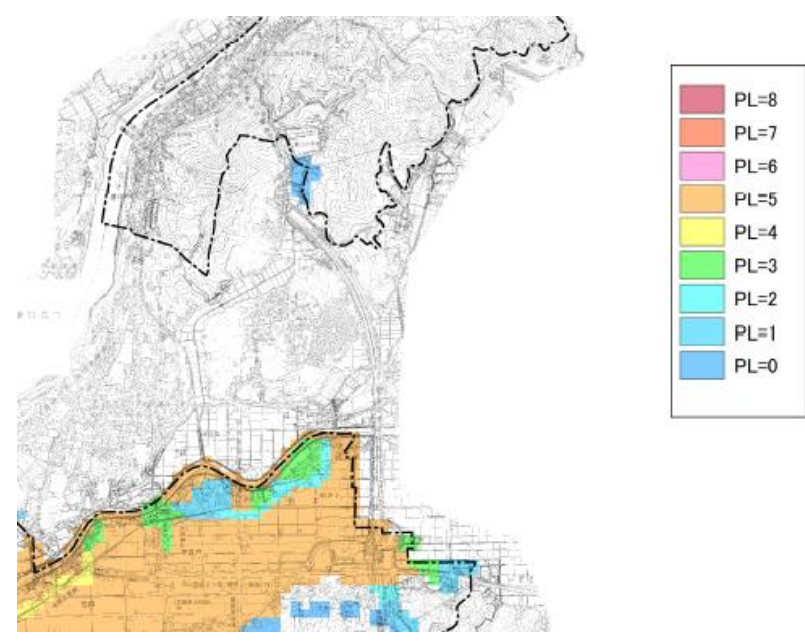

Figure 8. Example of excessive division in liquefaction risk. Although classes are separated according to $P_{L}$ value, it is difficult to match the legend to the map because the many classifications.

\subsubsection{Specific area is not evaluated nor color-coded}

Figure 9 shows an example of a map that leaves out a specific area: the industrial area is not color-coded nor evaluated, despite the possibility of liquefaction. In this case, users cannot determine the risk of liquefaction for the local community center, which may be utilized as an evacuation center during disaster.

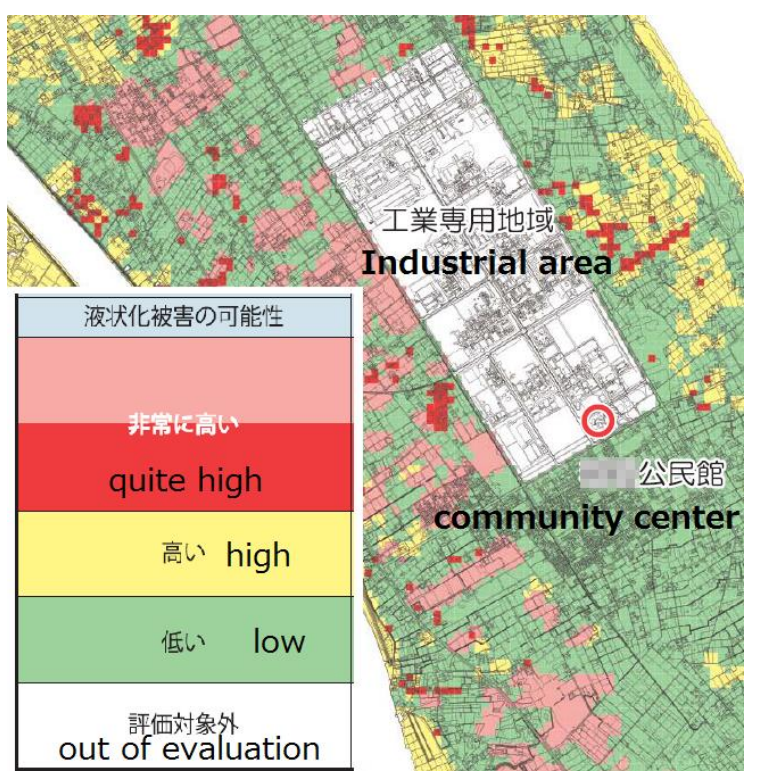

Figure 9. Example of a map lacking sufficient color-coding. Risk assessment has not been done in the area labelled as "Industrial Area," an area that includes the community center. 


\section{Discussion}

\subsection{Use of color to express liquefaction risk}

Our cluster analysis found that most liquefaction hazard maps investigated in this study use one of the following patterns of colors: (1) sequential color schemes that include red, yellow, and green (or blue); (2) warm color schemes that include colors such as red (purple), orange, and yellow; or (3) cold color schemes that include colors such as blue, cyan, and achromatic shades. Of these sequential color schemes are most similar to the safety color scheme as defined by Japanese Industrial Standards (JIS) where red means danger, for example. We found that many maps use this same color scheme, and it is a common tool for expressing liquefaction risk in Japan.

Previous studies have found that red and green color combinations should be avoided in risk maps (e.g. Livingstone and Hubel, 1988). Of those maps in Cluster 1 including these two colors, more than $60 \%$ had a minimum color difference $\Delta E_{00}$ under 10 . This seems to agree with previous studies. However, maps in Clusters 4 and 5, which included combinations of colors other than red and green, had an even higher proportion of maps was with $\Delta E_{00}$ less than 10 , implying that there are many other color combinations that may be difficult for people with colorblindness to distinguish between. Map developers should be aware of these possible accessibility issues and take care when creating hazard maps.

Finally, as demonstrated by Figure 2, no map should include more than 6 colors. However, because many maps include colors for purposes other than liquefaction risk, the total number of color classes in the legend should be limited to 3 or 4 .

\subsection{Color scheme variation and possible issues}

In Japan, the creation of liquefaction hazard maps is mainly based on the following resources: "Manual for Liquefying Area Zoning" (Earthquake Disaster Countermeasure Division, Disaster Management Bureau, National Land Agency, 1999), "Specifications for Highway Bridge, Part V Seismic Design" (Japan Road Association, 2002), prefectural earthquake damage survey reports, etc. (Une et al., 2018). These manuals include methods for evaluating liquefaction, making liquefaction distribution maps, and more. However, although they include some examples of maps, they do not include any information about composition, such as color scheme or color universal design. This means that each local government decides which color scheme and illustration style to use. As shown by the results of our color scheme survey, the methods of expression and contents vary greatly.

\subsection{Improving hazard maps}

As a result of the wide variety in hazard maps, there can be regional differences in risk perception. In addition, residents near a municipal boundary may find it difficult to access and/or understand both local governments' hazard maps: it is inconvenient to use similar hazard maps with different colors. In order to solve these problems, it is necessary to create a unified manual for hazard map creation. This should include information on proper color schemes, the utilization of a color universal design check tool, and the use of vector data with GIS.

\subsubsection{Verification of color scheme and color universal design}

We found many maps that had issues in color scheme and color universal design. To remedy this, we suggest that local governments rely on sources such as Ito (2012), Okabe and Ito (2008), and others. Such studies suggest color schemes, list caveats in the representation of diagrams, provide relevant checklists, and so on.

Because it is difficult for beginners to judge whether hazard maps satisfy color universal design using a checklist for each color, it is also useful to use auxiliary tools for color universal design, many of which now exist (e.g. Okabe and Ito, 2008; Jenny and Kelso, 2007). While these tools simulate the vision of a person with colorblindness, users must also note that a simulation will always differ from how a person actually experiences color. Despite this limitation, however, these tools can still identify colors that may be difficult for people to distinguish.

Integrating these processes into the creation of hazard maps will allow local governments to create maps that are easier to utilize and have an optimized number of color classes, hue and shading, and other elements (bordering, overlapping of colors, etc.).

\subsubsection{Utilizing digital data and GIS}

Our study included many images where the background map or hazard map itself seemed to be scanned to create electronic data. During the creation of a digital hazard map, if the background map must be based on a preexisting paper map, it is better to vectorize the scanned file or create new GIS data in order to prevent degradation of the resolution. The created digital data can then be used for both printing and online publication. Furthermore, creating a legend at the same time as digitizing a map will prevent situations where the colors differ between the legend and the map: utilizing GIS makes it easy to select information and change colors.

\subsubsection{Creating maps for individual hazards}

In other cases, liquefaction hazard risk was displayed on the same map as other hazard risks. This may make it difficult to accurately grasp the risk of liquefaction at a specific point. Therefore, it is better to make individual maps for each hazard and include unique information such as risks and evacuation centers. A web-mapping system, such as a hazard map portal site, will enable these hazard maps to be linked despite remaining separate (Ministry of Land, Infrastructure and Tourism, 2018).

\section{Conclusion}

In this study, we focus on the use of color and accessibility of liquefaction hazard maps, summarizing some of the main problems and possible countermeasures. 
We found that those hazard maps rely on a variety of color schemes, the specific scheme does not influence the proportion of hazard maps with a minimum color difference $\Delta E_{00}$ less than 10 , a value which implies they are difficult to read for people with colorblindness.

We found that hazard maps were difficult to read for a variety of reasons. Some were blurry or had overlapping colors, for example. In order to solve these problems, it is necessary to create a unified manual that contains a summary of all relevant data needed to create accessible maps, including the examination of colors, the utilization of a color universal design check tool, and the use of vector data with GIS.

\section{Acknowledgments}

Liquefaction hazard maps were collected by Ms. Miharu TANAKA (Tohoku Univ. of those days), Mr. Ryusuke ANDO (Gaduate School of Osaka Univ. of those days) and Mr. Naoshi YONEKAWA (Chiba Institute of Science of those days) in practical training at the Geospatial Information Authority of Japan in 2016. We would like to express our deepest appreciation to them and the stakeholders of each university.

\section{References}

Alžběta, B., and Arzu, Z. (2017). The effect of spatial distance on the discriminability of colors in maps, Cartography and Geographic Information Science, 44 (3), 229-245.

Brewer, C.A. (1994). Colour use guidelines for mapping and visualisation. In: Visualisation in Modern Cartography (New York: Elsevier Science), 123-147.

Earthquake Disaster Countermeasure Division, Disaster Management Bureau, National Land Agency (1999): Manual for Liquefying Area Zoning (1998 F.Y. version) (in Japanese).

Ito, K. (2012). Color universal design: Towards barrierfree design for color visions. Journal of Information Processing and Management, 55(5), 307-317 (in Japanese with English abstract).

Japan Road Association (2002). Specifications for Highway Bridge, Part V Seismic Design (in Japanese).

Jenny, B., Kelso, N. V. (2007) Color design for the color vision impaired. Cartographic Perspectives, 57, 61-67.

Livingstone, M., and Hubel, D. (1988). Segregation of form, color, movement, and depth: anatomy, physiology, and perception. Science, 240, 4853, 740-749.

Luo, M.R., Cui, G., and Rigg, B. (2001). The development of the CIE 2000 colour-difference formula: CIEDE 2000, Color Research and Application, 26, 5, 340-350.

Ministry of Land and Infrastructure, Tourism (2018). Hazard map portal site (in Japanese). https://disaportal.gsi.go.jp/ (Last accessed on 12 November 2018).

Okabe, M., and Ito, K. (2008). Color Universal Design (CUD): How to make figures and presentations that are friendly to colorblind people. http://jfly.iam.utokyo.ac.jp/color/ (Last accessed on 12 November 2018).

Sharma, G., Wu, W., Dalal, E.N. (2005). The CIEDE2000 color - difference formula: Implementation notes, supplementary test data, and mathematical observations. Color Research and Applications, 30 (1), 21-30.

Touyama, H. (2005). Color Blindness and Dictionary. IPSJ SIG Technical Report, 105, 9-16 (in Japanese with English abstract).

Une, H., Komatsubara, T., Miyachi, Y., Nakano, T. (2018). Applying Earth Science to Mitigate Liquefaction Damage. Journal of Geography (Chigaku Zasshi), 127 (3), 423-438 (in Japanese with English abstract).

Yang, Y., Ming, J., Ming, Yu, N. (2012) Color image quality assessment based on CIEDE2000. Advances in Multimedia, vol. 2012, Article ID 273723, 6 pages. 